\title{
ENFASIS DE GERENCIA SOCIAL EN EL CURRICULO DE TRABAJO SOCIAL DE UNIMINUTO
}

\author{
Por: Olga Lucía Pérez Ruiz
}

\section{Resumen \\ El artículo aborda elementos generales que hicieron parte del Trabajo de Grado titulado "Énfasis de Gerencia Social en el Currículo de Trabajo Social", el cual fue presentando para optar al título como Especialista en Gerencia Social de la Corporación Universitaria Minuto de Dios. Incorpora a su vez aportes del enfoque de la Gerencia Social del Instituto Interamericano de Desarrollo. Principalmente, expone la correlación entre el Trabajo Social y la Gerencia Social, como fundamento para desarrollar el énfasis dentro de la carrera.}

Olga Lucía Pérez Ruiz es

Trabajadora Social, especialista en Educación y Desarrollo Cultural, especialista en Gerencia Social de Uniminuto y estudiante de Maestría en Educación de la Universidad de Los Andes, Docente Programa de trabajo Social Uniminuto.

\section{Abstract}

The article approaches general elements that were part of the graduation paper named, "Emphasis of Social Management in the Social Working Curriculum", which was presented to obtain the degree of Specialist in Social Management of the Corporation of the University Minuto de Dios. In turn it incorporates contributions of the Inter-American Institute of Development, in particular in the area of Social Management. The article mainly exposes the correlation between the Social Working and the Social Management as groundwork in order to develop this theme throughout the degree. 
La importancia de haber realizado la Especialización en Gerencia Social que ofrece la Facultad de Ciencias Económicas y Administrativas de la Corporación Universitaria Minuto de Dios, se fundamenta en el propósito académico como docente de Trabajo Social, de abordar el conocimiento de dicho campo, en lo que respecta a sus fundamentos teórico conceptuales y las herramientas que sustentan su implementación. intuitivamente, se puede plantear un significado de la Gerencia Social y se pueden designar una serie de asignaturas que parecen relacionarse con tal concepto; sin embargo, cuando se habla de un énfasis o de un enfoque en el contexto de un currículo académico, es necesario saber de qué se está hablando, y con ello, de qué manera va a ser evidente en el plan de estudios, en las estrategias pedagógicas, en la investigación, en la proyección a la comunidad y en la práctica preprofesional. Como se expresa en el documento "Fundamentación Teórica y Estructura Curricular" del Programa de Trabajo Social (2000), la proyección social es el eje articulador de la Universidad Minuto de Dios, y responde a su origen fundacional. Este componente fuerte en lo social, llevó a la necesidad de incluir dentro de la oferta de formación profesional la carrera de Trabajo Social.

Otro factor relacionado con la creación del Programa, es la tradición de Trabajadores Sociales que han estado al frente en la gestión de programas y proyectos sociales en la Obra Minuto de Dios, a lo largo de cincuenta años. La Universidad por tanto, quiso reto- mar esta experiencia y estructurar un plan de formación para ofrecer a la sociedad nuevos Trabajadores Sociales.

En el documento mencionado, aparece estructurada la propuesta de formación, con su debida justificación, los propósitos académicos, los objetivos de formación, el perfil profesional y ocupacional, las competencias profesionales que se esperan desarrollar en el estudiante, aspectos pedagógicos generales, la práctica, la investigación, las estrategias metodológicas, el plan de estudios, los núcleos de conocimiento, los recursos y la evaluación.

La oferta de formación gira en torno a núcleos de conocimiento, en concordancia con los requerimientos del Consejo Nacional para la Educación en Trabajo Social CONETS, destacándose el interés particular de la Universidad por desarrollar competencias para la planeación y la gerencia social. Se ofrece la oportunidad de iniciar la Especialización en Gerencia Social durante el último año de la carrera. Además, se ofrece un área complementaria que es la financiera.

En el proceso de consolidación del programa, para el segundo semestre del año 2002, se confirman en la estructura curricular dos énfasis que son: la Gerencia Social y la Organización Comunitaria. Como oferta educativa, no se cuenta con una fundamentación teórico conceptual que argumente el por qué un énfasis en Gerencia Social; cómo se aborda en el currículo, qué beneficios y/o garantías 
ofrece al estudiante para su futuro profesional y qué indicadores nos van a permitir evaluar unos estándares de calidad en la formación. De igual forma, cómo va a realizar el curso de la especialización que se propone, y qué camino sigue hacia la formación postgraduada.

Partiendo de este planteamiento las preguntas que orientaron el trabajo de grado denominado “Énfasis de Trabajo Social en el Currículo de Trabajo Social de UNIMINUTO", fueron las siguientes: ¿Qué relación existe entre Trabajo Social y Gerencia Social? ¿Por qué la pertinencia de un énfasis en Gerencia Social para el Trabajo Social? ¿Qué implicaciones tiene el énfasis de Gerencia Social en el Currículo de Trabajo Social? .

El problema objeto se sitúa en el marco de la Gerencia Social como paradigma o enfoque contemporáneo de la administración y estilo gerencial modernos, lo mismo que en el marco de la Teoría Curricular, se aborda el currículo del actual Programa de Trabajo Social y de la Especialización en Gerencia Social. Se tienen en cuenta los marcos de referencia legal directos de la carrera de Trabajo Social, como son la Ley 53 de 1977 por el cual se regula el Código de Ética de la Profesión, y los Lineamientos para la Construcción de Programas Académicos de Trabajo Social dados por el Consejo Nacional para la Educación en Trabajo Social. Se analiza el contexto de la realidad colombiana y la urgencia de la Gerencia Social.
Las conclusiones y aportes del estudio, tan sólo se constituyen en la base para continuar madurando la realización del énfasis de Gerencia Social en el Currículo de Trabajo Social, con pertenencia social y pertinencia académica. Llama a la construcción participativa del currículo, con el concurso de los estudiantes y maestros, con quienes se podrá socializar el tema y analizar las estrategias para su incorporación en el mismo.

\section{Razones sociales para una formación en Gerencia Social}

En Colombia, como en el contexto general de América Latina, la lucha contra la pobreza es uno de los retos más importantes para el estado y la sociedad civil. La sociedad está afectada por una grave crisis económica y social que tiene efectos sobre el bienestar de la población. América Latina es la región del planeta donde el 5\% más rico tiene el mayor porcentaje de la distribución del ingreso y el $30 \%$ más pobre la menor. Se quiere demostrar que el tema de la pobreza y la desigualdad en nuestros países no es sólo un tema de falta de inversión y de capital; es también un problema de inequidad en la distribución de los ingresos y de exposición permanente de capitales hacia el exterior. (América Latina: una región en riesgo. Pobreza, inequidad e institucionalidad social. PNUD, Octubre de 1999).

Junto a la pobreza, la situación social de América Latina se singulariza por acentuadas 
Países más desarrollados, en términos de equidad (Suecia, Dinamarca, Países Bajos, otros)

\section{Países desarrollados}

\section{Gini promedio universal}

\section{América Latina}

inequidades. La región se ha convertido, según indican las cifras, en el Continente de mayor polarización social del mundo; es la región donde el $30 \%$ más pobre de la población recibe el menor porcentaje del ingreso $(7,6 \%)$ en relación a todos los otros continentes (BID). Medida asimismo en términos del coeficiente de Gini, que da cuenta del nivel de desigualdad en la distribución del ingreso de una sociedad, América Latina presenta el peor coeficiente.

Cuanto más bajo es el coeficiente de Gini, mejor es la distribución del ingreso en una sociedad. El de América Latina supera ampliamente a los de los países más equitativos, y es significativamente más elevado que la media mundial. Las acentuadas disparidades sociales de la región tienen impactos regresivos en múltiples áreas. Entre ellas: reducen la capacidad de ahorro nacional, limitan el mercado interno, afectan la productividad, tienen diversos efectos negativos sobre el sistema educativo, perjudican la salud pública, potencian la pobreza, favorecen la exclusión social, erosionan el clima de confianza interno, y debilitan la gobernabilidad democrática. (Klisberg. En: La Problemática de la Familia y la Educación en Améri-
0,25 a $\quad 0,30$ ca Latina, un Desafío Económico, Social y Ético).

Como lo explica Klisberg, las cifras generales de la pobreza se traducen en severas insuficiencias, en lo que el premio Nóbel de Economía en 1998, Amartya Sen, Ilama "capacidades de funcionamiento", es decir, las posibilidades que tienen las personas y las familias para funcionar adecuadamente en la sociedad; esto supone haber alcanzado determinados niveles de vida, tales como estados nutricionales adecuados, buenos estándares de salud y avances educativos. Se acude a la problemática del desempleo, para decir que este flagelo tiene efectos negativos sobre le bienestar y la libertad, lo cual va más allá de la pérdida de ingresos; incluye los daños psicológicos, la pérdida de motivaciones, de habilidades y autoestima, asimismo, aumento de enfermedades y mortalidad, ruptura de las relaciones familiares y sociales, acentuación de las tensiones raciales y las asimetrías de género.

Continúa el autor sustentando que enfrentar la pobreza requiere cambios de fondo en las políticas económicas y sociales de manera agresiva y eficiente. Por ello, destaca la nece- 
sidad de una Gerencia Social de buena calidad, con un abordaje diferente de las metas propuestas en el campo económico, ya que los objetivos que se persiguen no son alcanzables a corto plazo y además, son de orden cualitativo, deben generar la máxima participación de las comunidades implicadas y sentar las bases para una sustentabilidad.

Teniendo en cuenta que las causas de la pobreza son múltiples e interrelacionadas, Klisberg estima que lo social requiere abordar las problemáticas intersectorialmente, a través de un trabajo mancomunado de las diversas instituciones (Estado, sector privado, ONG's, comunidades de base) y la transformación hacia externalidades comunes. Deben darse estrechas alianzas donde se aproveche el potencial de cada participante. Con la intervención de cada sector por aparte, no se lograrán los cambios de fondo que se necesitan para hacer frente al problema estructural de la pobreza. Son necesarios los pactos y las concertaciones sociales de apoyo para lograr una gerencia social eficiente. Los programas sociales necesitan contar con respaldos amplios de la sociedad para poder cumplir con efectividad sus metas.

En lo social como en otras áreas del desarroIlo, la dimensión política es esencial. Las políticas públicas deben asumir la responsabilidad de garantizar los derechos básicos de trabajo, nutrición salud y educación a toda la población y una sociedad civil que movilice a fondos sus potencialidades de cooperar y participar en el esfuerzo necesario. Para ello es definitiva una buena gerencia social, frente a lo cual el papel de la Universidad cobra un rol muy importante en la formación de los futuros profesionales.

Klisberg plantea así, una “ ética de la urgencia “, ya que cada minuto que transcurre significa un grave deterioro de los niveles de vida de la población más vulnerable. Son de por sí, daños gravísimos, que en muchos de los casos son irreversibles.

\section{El significado de la Gerencia Social. La Gerencia Social como Paradigma}

Como lo expone la Trabajadora Social Julia Reyna de Zuluaga en su libro La Gerencia Social: Un Nuevo Paradigma en la Formación Profesional (1997), la Gerencia Social es un paradigma en tanto modelo de gestión de desarrollo y un patrón interno de dirección de las organizaciones que se estableció a partir de los diferentes paradigmas de la administración, de los cuales ha tomado los soportes para su aplicación.

Explica Reyna de Zuluaga que, a partir de los intentos encaminados a explicar el deber ser de la gerencia, se precisa una definición más amplia de la Gerencia Social: la Gerencia Social es un modelo de gestión que integra las antinomias del control normativo y el racional dentro de las organizaciones, es un sistema social centrado en la calidad, con estrategias de mercadeo, supervivencia y rentabilidad; modelo mediante el cual las organizaciones no gubernamentales y las 
dependencias estatales logren resultados en cuanto a las solución de problemas sociales y al mejoramiento de la calidad de vida en una economía reconocida en los años noventa como única e internacional .

De acuerdo con la autora, la Gerencia Social como paradigma, se enmarca dentro de nuevas lógicas las relaciones entre Estado, la sociedad y la empresa. El paradigma del Estado moderno ha creado la expectativa de resolver el problema de la pobreza. La construcción del futuro depende de la capacidad del Estado para modificar ambientes destrucción, ingobernabilidad y corrupción, y para gestionar opciones de vida a largo plazo. El futuro se construye teniendo en cuenta el contexto mundial y el margen de incertidumbre, evidente e indiscutible, dentro del cual se mueve la participación ciudadana en el mundo.

Como paradigma de gestión pública, la Gerencia Social involucra la participación de la empresa privada, las organizaciones no gubernamentales y los grupos comunitarios. El Estado tiene que gerenciar lo público, así como la empresa privada ha venido ejerciendo su gestión para garantizar sus intereses. Se plantea, entonces, un rediseño estructural y del Estado, de acuerdo con el modelo de Gerencia Social y dentro del proceso de revolución del conocimiento, la tecnológica, la informática y la política.

La Sociedad Civil, con derechos y deberes, impone al Estado, a partir de la carta consti- tucional, la nueva responsabilidad de una política social y asimismo, impone un nuevo gobierno en el que se incorporan grandes sectores de población. Dentro del Estado moderno la participación se convierte un mecanismo para sustentar el poder.

La experiencia internacional muestra que el trabajo conjunto del Estado y la sociedad es el que ha producido mayores logros. De tal modo que, bajo el nuevo paradigma, el Estado actúa junto con la sociedad; a diferencia de lo ocurrido bajo el paradigma de lo tradicional, en que Estado y sociedad se oponen. Las organizaciones bajo el paradigma de la gerencia social, están centradas en la responsabilidad; la gerencia establece límites respecto al ejercicio del poder y mecanismos de control interno, de acuerdo con las responsabilidad social adquirida en el contexto de las demás organizaciones de la sociedad. (60-63)

\section{Gerencia Social, Servicios Sociales y Bien- estar Social}

Por su parte, La Trabajadora Social María Lorena Molina (1996) desarrolla la teoría de la Gerencia Social en relación con los Servicios Sociales y el Bienestar Social, destacando el papel que cumple el Trabajador Social en la planificación, producción y distribución de tales servicios. (Revista Colombiana de Trabajo Social No.9. p.13-17)

La Gerencia Social es una disciplina científica, es técnica y es un arte derivado de las Ciencias Sociales y de la Administración, cuyo objeto consiste en estudiar la producción y 
distribución de los servicios sociales, en tanto éstos son la traducción de las políticas sociales en programas. Así, los sistemas de producción y distribución de servicios, son los principales responsables de la Gestión de las Políticas Sociales enmarcadas en una perspectiva humanista del desarrollo social.

Al referirse la Gerencia Social a la Producción de Servicios Sociales, ésta se conceptualiza como la relación demanda/ planificación y ejecución de los procesos de trabajo/ producto. La distribución de los servicios sociales comprende por su parte, los mecanismos establecidos para identificar la población de cada servicio social, los criterios de clasificación, los procedimientos para el aprovechamiento del servicio, y la evaluación del resultado y del impacto del servicio.

La Gerencia Social tiene como objeto de estudio el campo de análisis de instituciones y la organización de la producción, la gestión y evaluación de los servicios y el impacto de éstos en el sistema social. Su objetivo es mejorar las políticas sociales y los sistemas de producción y distribución de los servicios sociales. Como disciplina y práctica, su finalidad se vincula al desarrollo integral del individuo y le progreso de la sociedad; para ello se inserta la temática del Bienestar Social, entendido éste como el estado de una población donde las necesidades o demandas fundamentales son satisfechas según las condiciones que la sociedad define como ideales, y la interpretación que de ellas hacen las élites gubernamentales.
Desde el punto de vista del bienestar, a la Gerencia Social le concierne la prevención de los problemas sociales y la atención de sus manifestaciones; por ello, tiene que ver con: la salud, la educación, la vivienda, la seguridad social, el empleo, entre otros.,y se ocupa de las demandas sociales de los individuos, las familias y grupos sociales.

Los servicios sociales tienen como funciones (Kahn, citado por Molina. P.15):

- Adaptación de la personalidad y aprovechamiento de las oportunidades para la sobrevivencia.

- Aprendizaje y reaprendizaje social tendiente a la superación de los errores.

- Organización y promoción comunitaria, procesos por los cuales una comunidad identifica sus necesidades, objetivos, los jerarquiza y manifiesta la voluntad de satisfacerlas, reuniendo los recursos y desarrollando actitudes y prácticas de cooperación y desarrollo.

- Regulación-control, función que permite a los organismos mantenerse dentro de cierto equilibrio y conservar un régimen determinado o modificar su funcionamiento para adaptarse a otra circunstancias.

El proceso de Gerencia Social se basa en el desempeño de las funciones claves del proceso administrativo con una perspectiva ecosistémica, socio-política y por tanto estratégica. El modelo dinámico de gestión de 
programas sociales recupera un enfoque participativo de la administración o gestión por objetivos, formulado desde 1954 por Peter Drucker; la teoría sistemas, la teoría de la organización, la teoría del conflicto y negociación, la planificación estratégica, la investigación-acción en el ámbito administrativo, la noción de calidad total de los procesos, y la participación ciudadana.

Para María Victoria Muñoz (1997), la Gerencia Social debe propender por articular las acciones dispersas del sector público y el sector privado en un sistema de Bienestar Social; sólo en este sentido se podrá pasar del individualismo altruista a la responsabilidad social frente la desarrollo. El sistema podrá así trabajar con principios de solidaridad, cooperación, y subsidiaridad entre los actores que participan en el desarrollo. Muñoz agrega que es una tarea del estado y de la sociedad civil trabajar en estrategias que permitan la organización de un trabajo interinstitucional e intersectorial. El sistema de Bienestar Social logrará de esta manera esa integración orgánica que la modernidad plantea: la transformación del hombre y la sociedad a través también de las acciones institucionales. Desde esta perspectiva, la Gerencia Social permite pasar del interés particular al interés colectivo, y por tanto a las metas de desarrollo social.

\section{La Gerencia Social y las Alianzas}

La Gerencia Social constituye un pensamiento estratégico para la construcción de alianzas, necesarias entre sectores que han estado actuando en la sociedad en escenarios distantes unos de otros por sus intereses particulares. Aporta así, elementos para la información y la participación de los distintos actores en los procesos de planeación participativa frente al desarrollo. El fortalecimiento institucional del sector público y privado permitirá una participación racional reflexiva de compromisos de impactos y acciones eficientes en las tareas del desarroIlo. (Muñoz, María Victoria. 1997)

De esta manera, las Alianzas permiten el diálogo entre el sector público, el sector privado y las organizaciones civiles hacia el logro de un bien común. Las Alianzas por tanto:

- $\quad$ Fortalecen el Tejido Social: Crean una nueva instancia para la interacción de las personas y las organizaciones de sectores de la sociedad que, históricamente han tenido muy limitado contacto. A partir de esta instancia, se construyen relaciones más sólidas y confiables.

- Aumentan la cantidad de recursos disponibles y mejoran La capacidad de dar respuesta institucional (Flórez, Margareth. et al, 2002. 6) .

Las alianzas potencian las fortalezas de cada uno de los socios, disponen para el diálogo entre actores, agregan valor a lo que cada uno sabe hacer mejor y entregan resultados 
concretos y significativos; de ahí que se dice, mejoran el aprendizaje social. Tienen capacidad de Sostenibilidad ante la amenaza de cambios políticos e institucionales. Son democráticas y horizontales, independientemente del volumen o de la calidad de la contribución individual que los socios hagan. A pesar de las múltiples ventajas de trabajar en alianza, no todas las logran desarrollar las ventajas mencionadas, pues dependen de los factores del contexto social, político, cultural y económico, así como de la claridad estratégica con que opere la alianza y el compromiso de los socios, entre otros aspectos. Sin embargo, pueden mencionarse los beneficios que para los distintos sectores trae esta estrategia de desarrollo (6):

Para el sector público.

- $\quad$ Agilidad y flexibilidad en la solución a problemas de la comunidad

- Oportunidad de innovar

- Sostenibilidad de los programas.

- Oportunidad para implementar nuevas formas de gobernabilidad, que permiten al gobernante incorporar la participación de otros actores en la atención de asuntos públicos y ganar mayor confianza y credibilidad frente e su comunidad.

Para el sector privado.

- Reconocimiento por su aporte al desarrollo social.

- Lealtad de parte de la comunidad hacia la empresa.

- Un entorno social y económico mas saludable para el desarrollo de su misión empresarial.

- Una visión mas amplia del entorno donde se desarrolla su negocio

- Nuevas capacidades y habilidades para los miembros de la empresa involucrados en la alianza, las cuales probablemente no seria adquiridas si se estuviera exclusivamente en le ambiente de trabajo empresarial.

Para las organizaciones civiles.

- Oportunidad para ampliar el impacto en sus acciones

- Mayor disponibilidad de los recursos

- Reconocimiento frente a la comunidad y ante otros actores sociales.

\section{La Gerencia Social frente al Estado y la So- ciedad Civil}

Siguiendo a Ma. Victoria Muñoz, en su análisis sobre el Desarrollo Social, La Gerencia Social en las instituciones del Estado, busca que mediante un ordenamiento administrativo gerencial tengan éstas más gobernabilidad que mediante una posición filosófica y política, y ordenen sus acciones hacia objetivos comunes sociales, logrando así una legitimidad ante la sociedad. Por esto es necesario reestructurar y redefinir el papel de las instituciones del Estado más allá de los intereses partidistas y burocráticos. El proceso de modernización del Estado parte de la implementación de procesos democráticos como formas de organización social; en este sentido se debe entender que 
este proceso no es sólo una modernización institucional de orden administrativo, es fundamentalmente el cambio de mentalidad frente a lo que significa el servicio público, lo cual exige un cambio de mentalidad desde el alto gobierno, en el sentido de que las acciones del Estado no son solamente deberes, sino también derechos de los ciudadanos. Así cobra importancia la participación en los procesos de planeación, mediante la opinión y la iniciativa en los distintos ámbitos territoriales para que dichos planes respondan real y efectivamente a los intereses de los ciudadanos.

El papel de la Gerencia Social frente a la sociedad civil, es buscar que sus instituciones encuentren en el escenario nacional un posicionamiento y visibilidad de su trabajo; para ello se hace necesario consolidar y legitimar su papel en el proyecto de desarrollo social, a través de su organización política. (84)

Ese escenario de posicionamiento se instala en lo Local, escenario definitivo para una real participación. Estamos ante una nueva concepción del mundo como aldea global que muestra la emergencia de una escala regional poblada de nuevos agrupamientos territoriales; lo local surge entonces, como un nuevo espacio de articulación entre lo globalregional. Este giro hacia lo local requiere la construcción de escenarios que creen las condiciones necesarias para un nuevo tipo de participación social. El proceso de descentralización y el avance de la sociedad civil organizada son factores que impulsan hacia una redefinición de las funciones y relaciones entre el Estado y la sociedad en donde nuevos actores adquieren nuevas funciones que conlleva a una profunda reforma institucional y una política coherente de formación gerencial ajustada a las características de cada instancia y a sus responsabilidades. (Oszlack Oscar. Estado y sociedad: las nuevas fronteras)

El análisis realizado con base en fundamentos teóricos sobre el tema, conduce a interpretar el significado de la Gerencia Social, de la siguiente manera:

- Nuevo paradigma de la administración y de los estilos gerenciales modernos, que conjuga a su vez estrategias de otras propuestas gerenciales modernas como son gerencia o administración estratégica, la reingeniería, la gerencia de servicios, la gerencia de proyectos, la gerencia integral, la calidad total, la gerencia del desarrollo, entre otras denominaciones.

- Es vaso comunicante para el mutuo entendimiento entre el Estado y la sociedad civil, en relación con el trabajo que hay que adelantar para lograr el desarrollo social y la superación de la pobreza. Esta interdependencia es viable mediante la conformación de alianzas y redes estratégicas, es decir, el trabajo conjunto y asociado entre los actores.

- Es la alternativa gerencial moderna, necesaria para ejercer un liderazgo participativo, ajeno a la corrupción y al clientelismo. 
- Es el estilo gerencial que debe asumir y ejercer el Estado en todas sus instancias nacionales y locales, llevando a la realidad el espíritu de participación a que insta la Constitución.

- Es el estilo gerencial que deben ejercer las organizaciones privadas, para permitir un trabajo conjunto entre sí mismas, y con las organizaciones de base y los organismos estatales a nivel local, regional y nacional.

- Es la Gerencia que pueden ejercer todos y cada uno de los ciudadanos, desde su profesión y trabajo, especialmente desde el gobierno local, y más, desde las organizaciones de base, de manera que los líderes de los sectores populares tengan la oportunidad de gerenciar sus propios recursos hacia el mejoramiento de sus condiciones y nivel de vida, mediante una acción política de participación.

- Es la Gerencia que comprende los derechos humanos, el conflicto y la paz como un diálogo de saberes; por tanto, propende porque dicho diálogo se vea materializado en acuerdos y alianzas desde la base, ya en el contexto comunitario como institucional.

- Es la Gerencia que conoce, interpreta, ejecuta y evalúa la realización de la política social frente a la superación de las situaciones carenciales y marginales de la población local, regional y nacional, a través de la construcción de indicadores sociales y de impacto que se ven evidenciados en un balance social.
- Es la Gerencia cuyo valor ético es la Responsabilidad Social, la cual surge cuando cesa la relación contractual o legal; es decir, va más allá de la obligatoriedad o compromiso formal entre las partes, para abordar los intereses y necesidades cotidianos y tal vez ocultos de las personas, grupos y comunidades. La Responsabilidad Social, se entiende también como el compromiso con la comunidad del entorno, en sus aspectos medioambientales y de respeto por los espacios públicos.

- Es la Gerencia que promueve una alta participación y motivación de las personas, las instituciones y las comunidades en las decisiones que les interesan o afectan.

Desde el enfoque del Instituto Interamericano de Desarrollo INDES, La Gerencia Social es un ejercicio que tiene lugar en entornos cambiantes, con múltiples lógicas, el cual supone superar el enfoque tecnocrático y tradicional en el diseño y formulación de las políticas sociales. Por tanto, se necesitan medidas proactivas para articular las políticas económicas, las políticas sociales y el desarrollo institucional, de tal manera que haya una entrega adecuada de los servicios sociales, esto es, con eficacia, eficiencia, equidad y Sostenibilidad. De ahí, que sea necesario la promoción del desarrollo económico y social con base en elementos éticos y axiológicos. 


\section{La Formación del Trabajador Social como Gerente Social}

Desde sus orígenes, el Trabajo Social como práctica de acción social y como profesión, se ha orientado a la satisfacción de las necesidades y a la búsqueda de soluciones frente a los problemas de individuos, grupos y comunidades, en un contexto geográfico, socioeconómico, ambiental, político y cultural determinado, caracterizado principalmente por el estado de vulnerabilidad de las mismas y la falta de participación tanto en los bienes y servicios que les corresponden, como en las decisiones que las afectan.

En este sentido, se ha considerado propio de la identidad profesional, el papel que cumple el Trabajador Social como mediador entre las necesidades del hombre y los recursos existentes para la superación de aquellas situaciones que obstaculizan su desarrollo y bienestar. El Trabajo Social, ha estado íntimamente ligado al fenómeno de la pobreza, conjuntamente con las funciones de administración y gestión de las entidades sociales, con una visión de justicia y con especial preocupación por las poblaciones vulnerables en el marco de la política social y del bienestar social. Así, los planes de estudio han venido incorporando la noción administrativa y gerencial de los servicios sociales, tanto sectoriales como polivalentes o de base, en todas las áreas comprendidas en el sistema de bienestar, como son Salud, Vivienda, Educación, Empleo, Recreación y Cultura, Seguridad Social; lo mismo que, abordando todo tipo de problemáticas y poblaciones tanto en el ámbito rural como urbano.

Su desarrollo y evolución como profesión, se sustenta en la forma como ha dado respuesta a las necesidades sociales. "Desde el asistencialismo, determinado por los países europeos, y el Trabajo Social adaptativo, surgido en Norteamérica, hasta el heterogéneo modelo de Trabajo Social en Latinoamérica, esta profesión ha estado articulada al cambio y al desarrollo". (Reyna de Zuluaga, 1997,110).

El Trabajo Social cumple funciones comprendidas dentro de la atención directa a individuos, familias, grupos y comunidades. Para ello, aprende métodos de intervención para abordar las situaciones de caso en el plano de una relación de ayuda terapéutica o de orientación y asesoría; como también, para trabajar con los grupos y comunidades tanto en relaciones terapéuticas, como de organización y fortalecimiento de la participación en función del bien común. Por otra parte, el Trabajador Social es un profesional que investiga los problemas sociales que obstaculizan el desarrollo armónico de estas instancias; propone alternativas de solución basado en el conocimiento y comprensión de la actualidad, de la política social, de los marcos teóricos y metodológicos contemporáneos, de las instituciones y organizaciones comunitarias. En este orden de ideas, desarrolla la capacidad de participar en la interlocución de los problemas que aquejan a la sociedad, especialmente los que tienen que ver con los 
sectores populares, para así, generar caminos de acción favorables a su bienestar integral. Un Trabajador Social, actúa como mediador entre las necesidades de la población y los recursos existentes para satisfacerlas, y la manera como se viabilizan mediante la formulación, ejecución y evaluación de las políticas y de los proyectos y programas sociales.

El Trabajador Social no es el "todero", como bien lo diría Ander Egg, pero sí el profesional que desarrolla un carisma especial para identificar las carencias y necesidades de las personas, ya individualmente o como parte de grupos o comunidades, en los diversos contextos culturales; las comprende y aborda multidimensionalmente, creando un clima de mutua confianza y apoyo permanente; orienta el clima organizacional hacia el mejoramiento en la relación con el cliente o usuario del servicio. El Trabajador Social es fundamentalmente sensible frente aquellas inquietudes y necesidades ocultas de las personas; tiene especial condición de entrega y disposición para interactuar con las familias aún en las condiciones más adversas.

Del Trabajador Social se espera, cumpla una acción política frente a las inequidades y la falta de participación real de las personas en todos los espacios de su intervención. No es un funcionario o empleado más; es un actor en la dinámica del conflicto, entendido éste, como la vida misma en la que convergen los diversos intereses y necesidades, las formas de ver, entender y asumir la realidad. La intervención se basa en la búsqueda de alternativas para la participación, por tanto, para la real inclusión en las decisiones que afectan a los individuos.

En la actualidad, al trabajador social se le plantea de acuerdo con las exigencias del paradigma de gerencia de los años noventa, una proyección ya no continental, sino mundial, de su profesión.(111)

"Es un deber ético, profesional y social, buscar formas innovadoras, creativas de aprovechar los recursos asignados para construir mejores propuestas de orden socioeducativo, terapéutico o asistencial, en la formulación, gestión y ejecución de las políticas sociales"(Molina, Ma. Lorena, 1996,10). Frente a este desafío, juega un papel importante la formación profesional recibida en el Pregrado. Señala Bernardo Klisberg (1983), que el perfil académico deseable para la formación del Gerente Social en el ámbito universitario, debe reunir entre otras las siguientes características:

- Formación Humanista y Científica

- Epistemología y metodología de la Investigación

- Comprensión histórico social del ambiente.

- Comprensión del ambiente organizacional

- Estudio del Estado y del sector público

- $\quad$ Formación de actitudes y aptitudes para la gestión democrática, con el cultivo de la criticidad - creatividad

- Desarrollo de conciencia y responsabilidad social 
- Combinar en la formación lo teórico con lo práctico

- Conocer los fundamentos de la Teoría de las Organizaciones y las Escuelas de Pensamiento Administrativo

Al abordar los aportes de cinco Trabajadores Sociales que son y se desempeñan como Gerentes Sociales:

- La Gerencia Social se enmarca dentro de unas nuevas lógicas de relaciones entre el Estado moderno - descentralizado, la sociedad y la empresa. El Estado moderno descentralizado es aquel que se coloca al servicio de los ciudadanos. Las instituciones deben igualmente ponerse al servicio de los ciudadanos, a través del trabajo conjunto con las demás organizaciones públicas y privadas, con base en procesos participativos y democráticos, construyendo redes y alianzas. (Rodríguez, Héctor Fabio. Gerente Social. COL Engativá).

- El Trabajador Social, en cumplimiento del rol como Gerente Social, debe ser formado hacia: una actitud proactiva dirigida al cambio; el pleno conocimiento de la dinámica sociopolítica y económica; la asimilación de los problemas como posibilidad de acción transformadora; la actuación ética por convicción en cumplimiento de su gestión; la capacidad política para controvertir y asumir los resultados de la controversia; entender que los recursos siempre serán escasos y que en sus manos está la responsabilidad de optimizarlos; asumir los riesgos aún a costa de equivocarse, siempre y cuando el error se tome como una posibilidad de crecimiento; la capacidad de liderar un trabajo conjunto con las demás organizaciones; la búsqueda y optimización de los recursos; el manejo de técnicas y herramientas gerenciales y de liderazgo.

- Para Héctor Rodríguez, el Trabajador Social es el profesional idóneo para ser y ejercer la Gerencia Social, dada la vinculación que ha tenido históricamente con la administración de los servicios sociales, el bienestar social y la política social. La Gerencia Social como nuevo paradigma y modelo de administración, le aporta la comprensión de las esferas pública y privada; el conocimiento del Estado; de la Democracia, de los Derechos y las Políticas Sociales; de lo local, regional, nacional e internacional; del trabajo en alianzas y redes; de las relaciones internacionales; de la concepción de ciudadano y ciudadanía. Una necesidad vital es hacer mayor énfasis en la evaluación de los procesos y el impacto que se generan para el cambio social.

Los entrevistados concuerdan en considerar que la formación en Trabajo Social es de por sí, un importante terreno abonado para asumir la Gerencia Social. De hecho, desde la profesionalización del Trabajo Social en el siglo XX, se le plantea como derrotero la participación en la construcción y formulación de la política social, como también la 
dirección y gerencia de los servicios sociales, que en últimas son el resultado de la aplicación de la política social. ¿Cuál es entonces el factor diferenciador al asumir la Gerencia Social?. El factor diferenciador, es en definitiva la posición política y gerencial que el Trabajador Social debe asumir frente ai cuadro de injusticia social colombiana, derivado de una inadecuada visión, direccionamiento y puesta en marcha de la política social, tanto desde el escenario privado como estatal.

La posición política se entiende como la capacidad crítica de interpretar la política pública en diálogo con la política de las instituciones privadas y del tercer sector, en cualquier ámbito de acción profesional en el que se desempeñe. El Trabajador Social como Gerente Social es un profesional activo, por tanto no pasivo, frente a la construcción del tejido social. Esto le exige una permanente lectura de las estructuras sociales, de la dialéctica entre lo cotidiano y lo institucional, para detectar inconsistencias, proponer y hacerse parte en procesos de cambio social. Ya desde el movimiento de la Reconceptualización (1965), propio de la historia del Trabajo Social, se planteaba la necesidad de formar nuevos Trabajadores Sociales, con conocimiento "real" de las propias necesidades "reales" de América Latina; con espíritu de lucha y reivindicación de las injusticias sociales; con vocación de inserción en las comunidades y con capacidad de construir nuevas propuestas alternativas ya en lo teórico como en la aplicación práctica de las políticas sociales.
La posición gerencial viene dada por la posición política de cambio desde el "ser", pero concretizada en una forma de "hacer" las cosas. Para su implementación en la realidad, la Gerencia Social propone una base de herramientas, las cuales deben ser utilizadas precisamente como "base". La construcción de nuevos caminos la fomenta el espíritu creativo y de avance de la posición política del Trabajador Social.

\section{Currículo, Formación y Énfasis.}

Sobre las anteriores consideraciones, cabe preguntar ?cuál es el papel de la formación universitaria?. Si partimos del concepto de formación, la Universidad debe propender por la integralidad del ser y su quehacer, por lo cual, desde la comprensión de un currículo holístico, integrador, sociocrítico, dicha formación no se va a limitar al estudio de una serie de asignaturas que hacen una sumatoria más no una sinergia. En el caso que ocupa, el Trabajador Social para que asuma su papel como Gerente Social, es necesario que se le forme como tal, a través de la estructura del diseño curricular, de manera intencionada y clara, lo que permita poder realizar la evaluación del proceso formativo. Los actores directos del programa académico (alumnos, docentes, directivos) deben conocer en qué consiste la gerencia social y de qué manera el currículo permite formar Trabajadores Sociales con un énfasis en Gerencia Social. Esta claridad, debe permitir un intercambio proactivo a nivel interdisciplinar, en tanto, el 
cursar una asignatura con otro programa, no implica que haya un diálogo de saberes, sino una oportunidad de adelantar en otro horario o de interactuar socialmente con otros diferentes a los de la carrera. Así, desde un enfoque de formación debe interactuarse con otros enfoques de formación, para construir nuevas estrategias para el cambio social. Así también, si no es claro el direccionamiento y las variables de la formación, ro se podrán establecer los indicadores de evaluación de dicha formación y su impacto en el medio. Los currículos integradores e integrados, desde una perspectiva holística, deben permitir generar propuestas concretas para la transformación de las condiciones estructurales que obstaculizan el desarrollo social. Así, el aprendizaje será "significativo" para el estudiante, motivándolo a construirse desde el primer semestre de la carrera, a tener claro un horizonte. Los docentes y directivos del programa académico por tanto, tendrán unicidad de criterios frente a la formación e igualmente, tendrán claro un horizonte. En este sentido, la investigación y el diseño de las prácticas preprofesionales tendrán una argumentación y unas políticas establecidas, compartidas por todos.

Partiendo de la ubicación de la Gerencia Social en el marco de los lineamientos del Consejo Nacional para la Educación en Trabajo Social, se encuentra que:

- Esta se ubica como función del Trabajador Social, entre otras, como son: promoción, prevención, educación, diagnóstico, terapia, rehabilitación, investigación, administración como corpus general de la formación, en los diversos campos donde se demanda el ejercicio profesional.

- Las Áreas de Formación por Núcleos y

Conocimientos que los apoyan, según el CONETS, y que son adoptados por el Programa de Trabajo Social de Uniminuto, guardan estrecha relación con el perfil académico deseable para la formación del gerente social en el ámbito universitario, que propone Bernardo Klisberg (citado por María Lorena Molina, 1996). 
- Enfoques cualitativos y cuantitativos de investigación social.

- El perfil profesional y ocupacional, por tanto, se relacionan directamente con los propósitos de la Gerencia Social. En el marco de las entidades públicas y privadas y las ONGs, el Trabajador Social, promueve y fomenta acciones de desarrollo y de bienestar social; planifica, gestiona y evalúa proyectos de desarrollo dentro de una concepción holística sostenible y participativa ; diseña y administra programas de gestión humana; participa en el diseño y la implementación de políticas sociales ,orienta, gestiona y coordina procesos preventivos, $y$ de acompañamiento con familias y grupos; orienta, asesora y gestiona procesos de desarrollo humano en organizaciones; participa en procesos de gestión regional y local y de cultura ciudadana.

- La Gerencia Social se presenta como conocimiento que apoya el núcleo denominado: diseño, gestión y evaluación de los Servicios Sociales. No se delimita necesariamente como asignatura.

Para terminar, es importante destacar el aporte de los Trabajadores Sociales entrevistados, quines consideran que el énfasis de Gerencia Social en el currículo de la carrera de Trabajo Social, debe estar orientado a la formación de profesionales con una visión crítica pero proactiva respecto a la realidad sociopolítica nacional, en un contexto mediado por acelerados procesos de globalización económica basada en el desarrollo tecnológico, ante todo se debe insistir en la dotación de herramientas conceptuales y metodológicas que permitan actuar en el campo profesional a partir del conocimiento que puede proporcionar el análisis y discernimiento permanentes.

El Trabajador Social con énfasis en Gerencia Social deber ser un investigador permanente, capaz de dudar de postulados aparentemente confirmados, debe ser un convencido de que lo único permanente es el cambio y que la dinámica social no se estanca como en ocasiones lo hace nuestro pensamiento 0 nuestro prejuicio personal; debe comprender que tiene en sus manos la posibilidad de ser un líder que puede incidir tanto en la dirección de microprocesos como en la construcción de un Estado democrático a partir de incidir en la formulación de la política pública con participación ciudadana.

No basta con obtener conocimiento sobre aspectos técnicos, metodológicos y económicos, si el Trabajador Social como Gerente Social no tiene competencia para dilucidar el contexto que le rodea y asumir una posición política, de análisis y lucha por la defensa de los derechos sociales; si no desarrolla habilidades de liderazgo que motiven a una real participación de las personas y grupos desde la base, lo mismo que desde las instituciones locales y regionales. Este planteamiento se da a la inversa, pues si no se aplican herramientas metodológicas y técnicas, de la mano de un proceso sistemático y organizado, la voluntad política de actuación se quedará en el discurso y la implementación intuitiva de la política social.(Rodríguez) 